\title{
VISUALIZATION OF LEAKY WAVES ON PLANAR TRANSMISSION LINES
}

\author{
Jan Machac*, Jan Zehentner*, Jan Mrkvica*
}

\begin{abstract}
This paper presents a visualization of leaky waves excited by delta-gap sources on planar transmission lines, namely the 2nd surface and space leaky modes on the slotline, and the dominant and surface leaky modes on the conductor-backed slotline. The field is modeled by numerical calculation in the time domain. The simulations show the character of the leaky waves excited by delta-gap sources and are a useful tool for the investigation of leaky waves.
\end{abstract}

\section{INTRODUCTION}

Leaky effects have been studied for more than forty years [1], with the aim to design leaky wave antennas. The behaviour of leaky waves on planar transmission lines has been intensively investigated since the 1980s [2-4]. The leaky waves cause various spurious effects on planar lines, such as increase of losses, crosstalk, and signal distortion.

Leaky modes can propagate under proper conditions on all open planar transmission lines. There are two kinds of leaky modes. Surface leaky modes divert energy into a substrate apart from the line axis. Space leaky modes take energy to the space above and if possible also below the line, and in most cases also into the substrate. Leaky modes, as the solutions of the wave equation when the line is infinitely long and the substrate also spreads to infinity, cannot be excited in their pure essence on a line fed by a real source of finite power and finite dimensions. The field of the surface leaky wave excited by a real source of finite power increases in the lateral direction to its maximum value, and then decreases in order to meet the radiation condition. The angle at which the field maximum can be detected corresponds to the leakage angle [3]. For weak leakage, when the leakage constant has a low value, this angle can be approximated by $\cos \Theta^{\prime}=\beta / k_{s}$, where $\beta$ is the leaky mode phase constant and $k_{s}$ is the phase constant of the substrate surface mode. The precise value of this angle has been determined in [5].

We studied the slotline (SL) and the conductor-backed slotline (CBSL), Fig. 1. Our conclusions are however generally valid and applicable to other planar transmission lines. We analyzed these lines by the method of moments modified as in the Galerkin testing procedure in the spectral domain with successive complex root searching (SDM). The visualization of the field of the leaky waves was performed by numerical modeling in the time domain by the CST Microwave Studio when the real source was represented by the delta-gap source and the substrate was terminated by absorbing layers. Numerical modeling can to some extent substitute experimental measurements. The advantage of numerical experiments is that the conditions under which the experiments are performed can be easily set in the electromagnetic simulator, e.g. an absorbing layer.

\section{SURFACE LEAKY MODES ON THE SLOTLINE}

We have revealed [4] and experimentally proved [6] the 2nd leaky mode (2nd LM) leaking energy simultaneously into $\mathrm{TM}_{0}$ and $\mathrm{TE}_{1}$ surface waves. This mode can propagate simultaneously with the 1st leaky mode (1st LM), leaking power only into the $\mathrm{TM}_{0}$ surface wave. The dispersion characteristics of these modes are plotted in Fig. 2. The 1st LM can propagate in the frequency band at which its phase constant is lower than $\mathrm{k}_{\mathrm{TM} 0}$. In the case of Fig. 2 at the frequencies higher than $6.1 \mathrm{GHz}$. The 2nd LM is physical and can propagate in the frequency band where its phase constant is lower than $k_{\mathrm{TM} 0}$ and at the same time lower than $k_{\mathrm{TE} 1}$. In Fig. 2 this frequency band starts from $4.9 \mathrm{GHz}$. The field of the $\mathrm{TM}_{0}$ surface mode is represented by the $E_{y}$ component, perpendicular to the substrate, whereas the field of the $\mathrm{TE}_{1}$ surface mode is represented by the $H_{y}$ component.

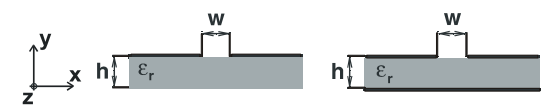

Fig. 1 Cross-sections of the SL and CBSL.
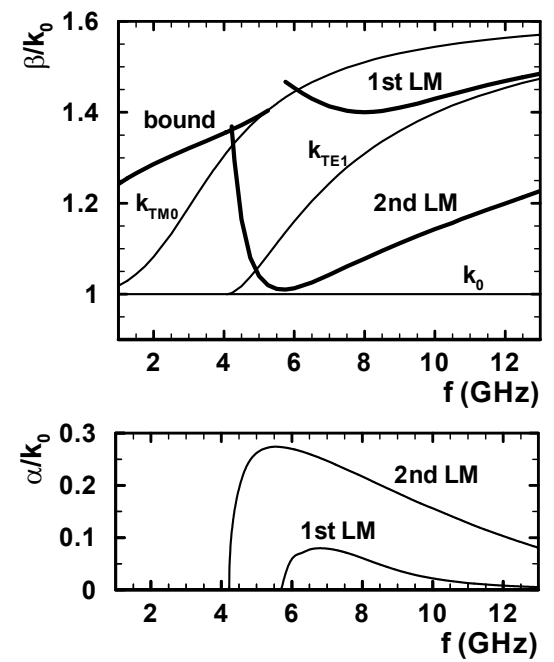

Fig. 2 Normalized phase $\beta / k_{0}$ and leakage $\alpha / k_{0}$ constant of modes on the SL with $w=5.65 \mathrm{~mm}, h=14.6 \mathrm{~mm}$, and $\varepsilon_{r}=2.6$.

\footnotetext{
* Czech Technical University, Technicka 2, 16627 Prague 6, Czech Republic, E-mail: machac@fel.cvut.cz
} 
In the numerical experiment the line was fed by a deltagap source of voltage $1 \mathrm{~V}$ connected across the slot on the top substrate surface. The calculated field distributions at frequency 10.75 GHz are shown in Figs. 3 and 4. Both the 1st LM and the 2nd LM can propagate simultaneously at this frequency, Fig. 2. Formula (7) in [3] gives at this frequency the leakage angle of the 1st LM $\Theta_{1}=21.5^{\circ}$. The 2nd LM is the superposition of $\mathrm{TM}_{0}$ and $\mathrm{TE}_{1}$ modes, which can be treated separately. The corresponding angles for these modes are $\Theta_{2 \mathrm{TM} 0}=42^{\circ}$ and $\Theta_{2 \mathrm{TE} 1}=36^{\circ}$. Fig. 3 shows that the distribution of the $E_{y}$ field is a superposition of two modes, the $1 \mathrm{st} \mathrm{LM}$ represented by the $\mathrm{TM}_{0}$ mode propagating at angle $\Theta_{1}=22^{\circ}$ obliquely to the line, and the 2nd LM represented by the $\mathrm{TM}_{0}$ part propagating at angle $\Theta_{2 \mathrm{TM} 0}=41^{\circ}$. The magnetic field $H_{y}$ representing the mode $\mathrm{TE}_{1}$ is plotted in Fig. 4, where it propagates at the angle $\Theta_{2 \mathrm{TE} 1}=32^{\circ}$.

\section{SPACE LEAKY MODES ON THE SLOTLINE}

The calculated dispersion characteristic of the 1st order space leaky mode (SLM) is shown in Fig. 5, together with the characteristics of the dominant and 1st order bound modes. Generally, the dispersion characteristic of the SLM is a multivalued function of the frequency. Only the first branch of the characteristic is shown in Fig. 5. The 1st order SLM is physical in the frequency range from 1.4 to $4.5 \mathrm{GHz}$, where the phase constant is lower than $k_{0}$. The amplitude of the 1 st order SLM grows to infinity in both the $y$ and $x$ directions. This however cannot be achieved when a real source supplying finite power is applied. The calculated field distribution of the 1 st order

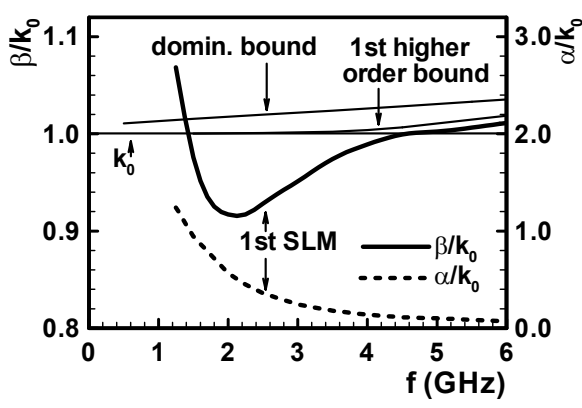

Fig. 5 Normalized phase $\beta / k_{0}$ and leakage $\alpha / k_{0}$ constant of the 1 st order SLM and the dominant and the 1st order bound waves on the SL with $\mathrm{w}=60 \mathrm{~mm}$, $h=1.2 \mathrm{~mm}$, and $\varepsilon_{r}=2.6$. SLM excited on the SL defined in Fig. 5 by the delta-gap source at frequency $3 \mathrm{GHz}$ is shown in Fig. 6. This corresponds to the description of the leaky wave presented in [1]. Leakage angle $\Theta_{p}=\operatorname{arcos}\left(\beta / k_{0}\right)=18^{\circ}$ fits with the angle $16^{\circ}$ at which the wave in Fig. 6 leaves the substrate. The angle of the

maximum far field radiation calculated by CST Microwave Studio is $21^{\circ}$, as read from the radiation pattern in Fig. 7. This angle represents the angle of the maximum of the unidirectional far field radiation $\Theta_{u}$ defined in [1]. These two angles $\Theta_{p}$ and $\Theta_{u}$ merge together with increasing frequency due to the fall of the attenuation constant, as was presented in [1]. The leakage angle decreases with increasing frequency, since the phase constant becomes higher. Angles $\Theta$ are measured in the SL symmetry plane from the forward direction.

\section{WAVES ON THE CONDUCTOR-BACKED LINE}

We have described and experimentally verified the dominant mode on the CBSL [7]. This mode can propagate from zero frequency up to the frequency at which the surface leaky

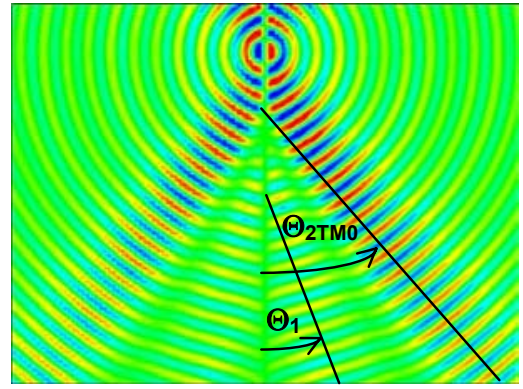

Fig. 3 Calculated electric field $E_{y}$ in the substrate of the SL with

$w=5.65 \mathrm{~mm}, h=14.6 \mathrm{~mm}$, and $\varepsilon_{r}=2.6$ in the plane $y=10 \mathrm{~mm}$ at the frequency $10.75 \mathrm{GHz}$. The edges of the substrate are terminated by absorbing boundaries to avoid any reflections.

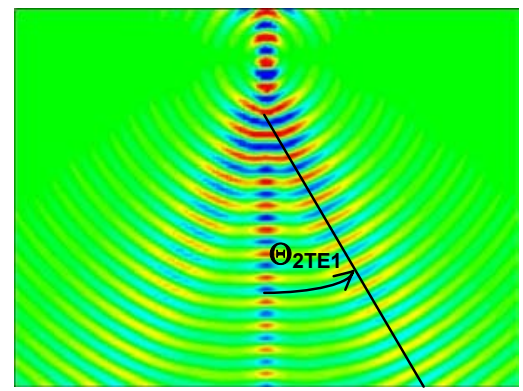

Fig. 4 The distribution of the magnetic field $H_{y}$ plotted under the same conditions as in Fig. 3.

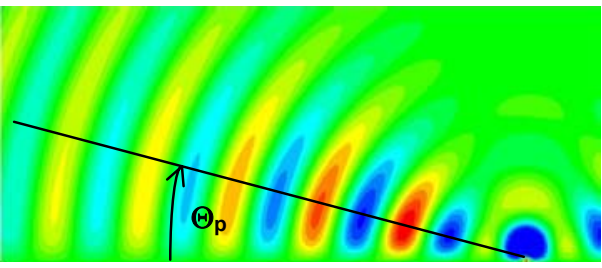

Fig. 6 Calculated electric field $E_{y}$ of the 1 st order SLM on the SL in the plane yz when $x=0 \mathrm{~mm}, \mathrm{w}=60 \mathrm{~mm}, h=1.2 \mathrm{~mm}$, and $\varepsilon_{r}=2.6$ at frequency $3 \mathrm{GHz}$. The edges of the substrate are terminated by absorbing boundaries.

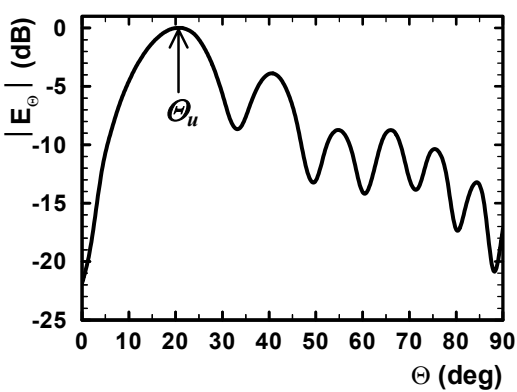

Fig. 7 Radiation pattern pertinent to the field distribution in Fig. 6 taken at the same plane as the field distribution in Fig. 6. 

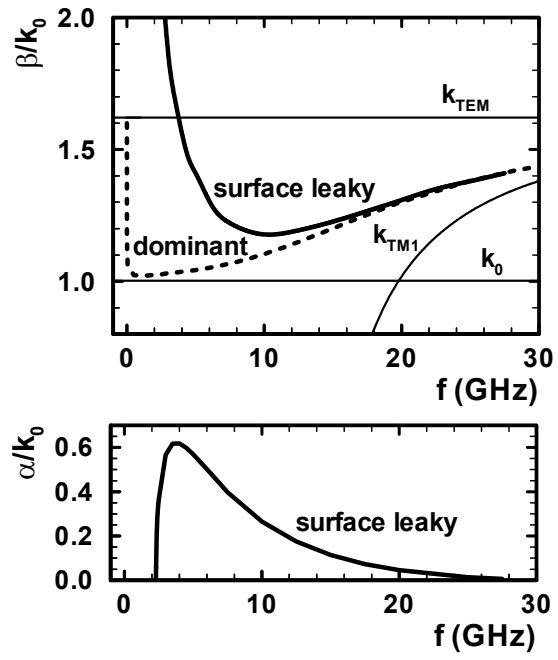

Fig. 8 Normalized dispersion characteristics of the even modes on the CBSL with $w=6 \mathrm{~mm}$, $h=6 \mathrm{~mm}$ and $\varepsilon_{r}=2.6$.

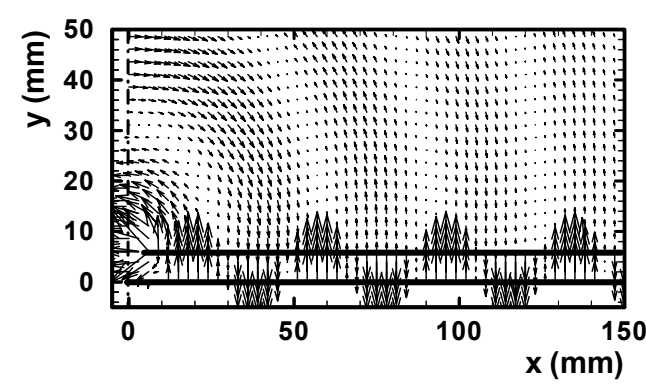

Fig. 10 The electric field distribution of the dominant mode on the CBSL defined

in Fig. 8 calculated by the SDM in the cross-sectional plane at $5 \mathrm{GHz}$.
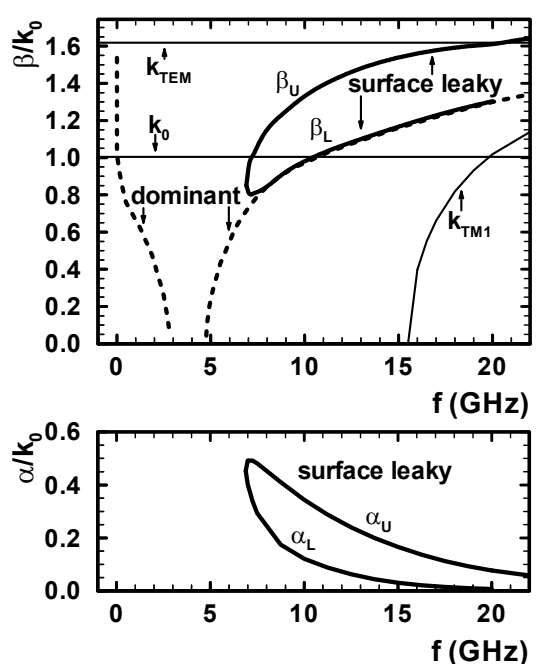

Fig. 9 Normalized dispersion characteristics of the even modes on the CBSL with $w=12 \mathrm{~mm}, h=6 \mathrm{~mm}$ and $\varepsilon_{r}=2.6$. mode is physical, as shown in Fig. 8. Above this frequency these two modes can propagate simultaneously. The dominant mode propagates along the line unattenuated and has the character of a standing wave in the lateral $x$ direction. This standing wave pattern can be interpreted as the superposition of two TEM waves propagating obliquely to the line axis at the angle $\Theta=\arccos \left(\beta / k_{0} \sqrt{ } \varepsilon_{r 2}\right) . \quad$ The dominant mode phase constant is lower than $k_{\mathrm{TEM}}$, the phase constant of the TEM mode in the dielectric substrate with double sided metalization, and can be even

lower than $k_{0}$, Fig. 9. Such a solution of the wave equation represents a mode which can potentially leak in space, as documented by the field distribution computed by the SDM in Fig. 10. The field in the space above the line decreases with distance, and its wavelength corresponds to the propagation constant $k_{y}=\sqrt{ }\left(k_{0}^{2}-\beta^{2}\right), \lambda_{y}=2 \pi / k_{y}=66.5 \mathrm{~mm}$. The propagation of this pure dominant mode is possible only on an infinitely long line on an infinitely wide substrate. The source exciting it must supply infinite power and be distributed over the whole infinite substrate width. In spite of exciting the field by a real source and imperfect suppression of reflections from the substrate edges, we were able to reveal the standing wave pattern of the dominant mode in the measured field maps taken on the front substrate edge [7]. A numerical experiment makes excitation of the

dominant mode possible at least in a restricted area. On the other hand it turned out that this wave cannot be excited by a delta-gap source, since the field is not coupled to the line and escapes into the substrate and into the space. Fig. 11a shows the simulated electric field in the substrate of the CBSL with $h=6 \mathrm{~mm}$, $w=6 \mathrm{~mm}, \varepsilon_{r}=2.6$ when a TEM wave is incident at the angle $\Theta=\arccos \left(\beta / k_{0} \sqrt{ } \varepsilon_{r 2}\right)$ to the region below the slot. The source is uniformly distributed along the top edge of the substrate. The substrate edges are terminated by absorbing boundaries to protect the field from reflections. The field reflected on the nonhomogeneity represented by the slot is superimposed on the incident wave and results in the wave propagating along the slot.

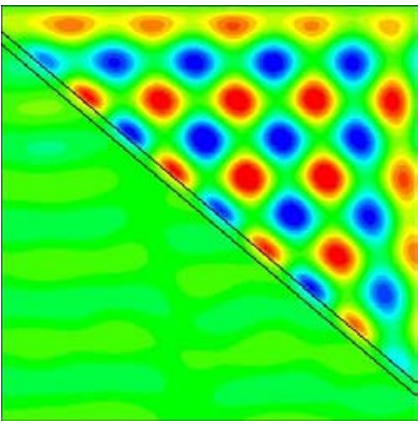

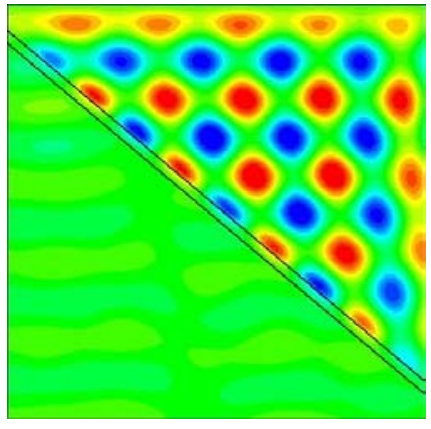

b

Fig. 11 Simulation of the even dominant mode on the CBSL with $h=6 \mathrm{~mm}, w=6 \mathrm{~mm}, \varepsilon_{r}=2.6$ at $5 \mathrm{GHz}$, (a) $E_{y}$ field in the substrate., (b) $E_{y}$ field in air above the slot.

Laterally it has the character of a standing

wave. The field does not pass to the area on the opposite side of the slot but is coupled though the slot to the air, where the wave propagating along the slot appears as shown in Fig. $11 \mathrm{~b}$. Its wavelength is $\lambda=2 \pi / \beta$. The same effect occurs when discrete delta-gap sources are placed between parallel plates, perpendicular to the line axis, spaced periodically with an alternated adjoining phase shift, Fig. 12. The dominant wave, which has laterally the character of a standing wave, is here observable in the area of the triangle with the base at the top of the plot where the sources are located. The wave travels from the top downwards. 
The dispersion characteristic of the surface leaky mode on a CBSL with a wider slot is an ambiguous function of frequency, Fig. 9. The phase constant can fall below $k_{0}$ or can in the case of the upper branch be greater than $k_{\text {TEM }}$. At these frequencies the corresponding mode is nonphysical. Numerical experiments show that the surface leaky wave can be excited on the CBSL as a complex wave propagating along a line with decreasing amplitude. At frequencies where the two modes corresponding to the two branches of the dispersion characteristic are physical we are able to distinguish in the plot of the simulated field the wave guided along the line with the wavelength corresponding to $\left(\beta_{L}+\beta_{U}\right) / 2$. This is the phase constant of the superposition of the two waves under the assumption of the same amplitudes with phase constant $\beta_{L}$ of the lower branch and $\beta_{U}$ of the upper branch, Fig. 9. At frequencies where only one mode is physical the wavelength is referred to the phase constant of this mode.

\section{CONCLUSION}

The paper presents numerical experiments visualizing the behaviour of the leaky waves excited by delta-gap sources on the SL and the CBSL. They emulate the measurement, supplement the investigation of the leaky waves, and provide a real picture of their fields.

The distribution of the electric and magnetic fields

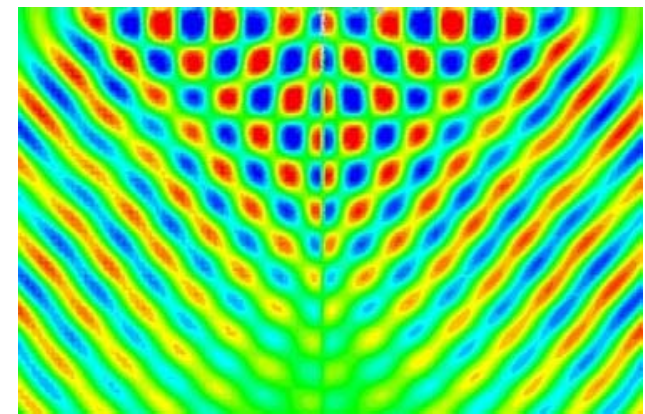

Fig. 12 Excitation of the even dominant mode on the CBSL by an array of deltagap sources connected across the substrate edge sequentially with alternating phase, $h=6 \mathrm{~mm}, w=6 \mathrm{~mm}$, $\varepsilon_{r}=2.6,5 \mathrm{GHz}$. calculated by the CST Microwave Studio confirms that the 2nd surface leaky wave is the superposition of the $\mathrm{TM}_{0}$ and $\mathrm{TE}_{1}$ surface waves propagating in the substrate at the angles defined by the formulas derived in [5]. This wave can propagate simultaneously with the 1st surface leaky wave. These two waves are well distinguished in the presented plots. The numerical simulation of these waves excited by a delta-gap source connected across the slot confirms the results of the measurement done in [6]. The numerical simulation of the space leaky wave excited by the delta-gap source on the slotline with the wide slot confirms the theoretical assumption that the wave leaves the substrate at the angle determined by the parameters of the pure space leaky mode calculated by SDM on the infinite structure.

Numerical experiments enable simulation of the dominant wave on the CBSL at least in a restricted area using a source distributed uniformly along the substrate edge, which releases the wave incident obliquely to the slot. The dominant wave can alternatively be excited again in a restricted area by an array of delta-gap sources spaced periodically with an alternated adjoining phase shift. In addition numerical experiments confirmed that the wave propagating along the CBSL with a wide slot is the superposition of the two surface leaky modes which correspond to the two branches of the dispersion characteristic.

\section{References}

[1] T. Tamir, and A. A. Oliner, "Guided complex waves Part 2. Relation to radiation patterns," Proceedings I.E.E., vol. 110, pp. 325-334, Feb. 1963.

[2] A. A. Oliner, "Leakage from various waveguides in millimeter wave circuits," Radio Science, vol. 22, pp. 866-872, Nov. 1987.

[3] H. Shigesawa, M. Tsuji, and A. A. Oliner, "Theory and experiments of simultaneous propagation of both bound and leaky dominant modes on conductor-backed coplanar strips," Proceedings of the 23rd European Microwave Conference, Madrid 1993, pp. 1005-1008.

[4] J. Zehentner, J. Machač, and M. Migliozzi, "Upper cutoff frequency of the bound wave and new leaky wave on the slotline,“ IEEE Trans. Microwave Theory Tech., vol. 46, pp. 378-386, Apr. 1998.

[5] J. Macháč, and J. Zehentner, "Comments on representation of surface leaky waves on uniplanar transmission lines," IEEE Trans. Microwave Theory Tech., vol. 50, pp. 583-585, Feb. 2002.

[6] J. Zehentner, J. Machac, J. Mrkvica, M. K. Sarnovski, and J. Polivka, "Surface leaky waves and their measurement," Proceedings of the 28th European Microwave Conference, Amsterdam 1998, vol. 2, pp. 561564.

[7] J. Zehentner, J. Machac, J. Mrkvica, and C. Tuzi, "Modes on the standard and inverted conductor-backed slotline,” 2003 IEEE MTT-S Int. Microwave Symp. Dig., June 2003, vol. 2, pp. 677-680. 